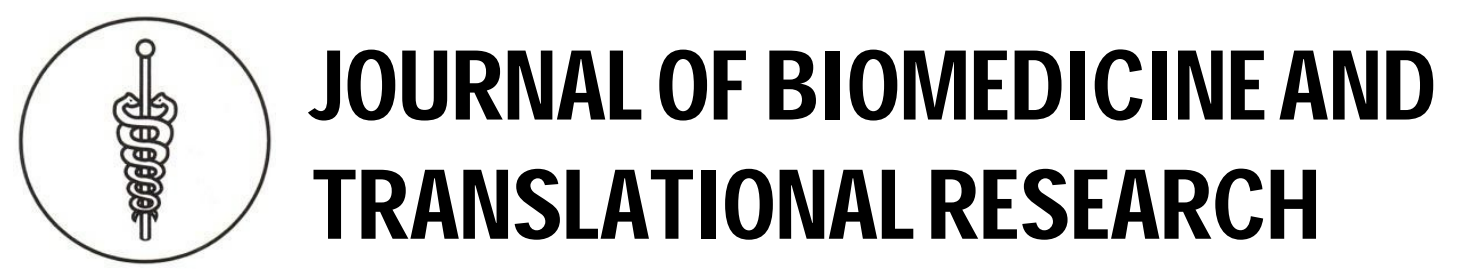

Copyright $\mathrm{C} 2016$ by Faculty of Medicine Diponegoro University and Indonesian Doctor Association, Central Java Region

\title{
Macrophage Activity Test Of Pulmonary Tuberculosis Patients With Diabetes Mellitus (TB-DM)
}

\author{
Antari Arlita Leniseptaria ${ }^{1}$, Indah Saraswati ${ }^{2}$, David Pakaya ${ }^{3}$, Aryoko Widodo ${ }^{2}$ \\ 1. Department of Microbiology Faculty of Medicine Diponegoro University \\ 2. Department of Chemistry Faculty of Medicine Diponegoro University \\ 3. Department of Histology Faculty of Medicine and Health Science Tadulako University
}

\section{Article info}

History :

Received 1 December 2016

Accepted 15 December 2016

Available 29 December 2016

\begin{abstract}
Background: Control of pulmonary TB is getting more and more complicated as the number of patients with diabetes mellitus (DM) is increasing. The increasing prevalence of DM is followed by the increasing prevalence of pulmonary TB. Diabetes Mellitus patients have 4,7 times higher risk to develop pulmonary TB compared to patients without DM, since DM can increase the frequency and severity of an infection, including pulmonary TB. Aim: To analyze macrophage activity (phagocytosis, intracellular killing, and TNF- $\alpha$ synthesis) of TB-DM patients.

Method: The research was an experimental study used a PBMC cultured sample from TBDM patient's which undergo observation of macrophage activity (phagocytic, intracellular killing and TNF- $\alpha$ synthesis). The data were taken from microscopic observation of TBDM patients, colony growth of viable Mycobacterium tuberculosis and the TNF- $\alpha$ level secreted by macrophages.

Result: Microscopic observation showed that there wereless amount of phagocytosed $M$. tuberculosis (in macrophage/intracellular level) and there was a little amount of formed vacuoles and giant cells. Furthermore, macrophages in TB-DM patients secrete low level of TNF- $\alpha$, and there were more viable $M$. tuberculosis from this macrophage.

Conclusions: Macrophages of TB-DM patients are less activated, with reduced phagocytic activity toward M. tuberculosis..
\end{abstract}

Keywords: macrophage, TB-DM, phagocytosis, intracellular killing, TNF- $\alpha$.

\section{INTRODUCTION}

Tuberculosis (TB) is a major cause of death among bacterial infection diseases. TB affects 9,4 million people and kills 1,7 million of world population annually. The etiology is Mycobacterium tuberculosis bacteria which has a rod shape, aerobe characteristic, and acid-fast feature. Indonesia rank 4th worldwide for its high number of TB patients 1,2,3 World Health Organization expects that TB control will become more difficult with the increasing number of diabetes mellitus (DM) patients, due to DM is one of the risk factors for TB deterioration.
Correlation between TB and DM have been reported since $1000 \mathrm{AD}$, though it is still difficult to be defined. ${ }^{4,5,6}$ The increasing cases of TB-DM are associated with an increase in morbidity and mortality of TB and DM. DM patients have ${ }^{4,7}$ times higher risk to develop pulmonary TB. ${ }^{4}$ This is due to the treatment of MDR-DM cases, which one of its aim is to restore the function of the immune system, i.e. immunostimulant. ${ }^{7}$ Less activated alveolar macrophage of pulmonary TB patients with DM reduces the interaction between $T$ lymphocyte and macrophage, resulting in defect of M. tuberculosis elimination. 
The entry of $M$. tuberculosis into the macrophage and its ability to survive are the key element of the pathogenesis of tuberculosis. ${ }^{8,9} \mathrm{On}$ primary infection, aerosol droplet nuclei containing M. tuberculosis is inhaled and settle on the pulmonary alveolar epithelial cell surface expressing adhesion molecule (intracellular adhesion molecule-1/ICAM-1), thereby increasing the migration and adhesion of phagocytic cells, particularly alveolar macrophage which effectively phagocyte all particles including $M$. tuberculosis. ${ }^{9,10}$ Immune response by macrophage in the form of phagocytosis and intracellular killing which contributes to the immune defense is expected to be the first in line to eliminate $M$. tuberculosis and lower the incidence of TB. However, M. tuberculosisis able to multiply within macrophage thereby causing tuberculosis.

The increase in cases of TB-DM that associated with the increased of morbidity and mortality due to TB-DM, which is DM patients have an immune respond disorder, thereby facilitating $M$. tuberculosis infection. Thus, it is important to know more about the treatment of cases of TB$\mathrm{DM}$, one of which emphasizes the restoration of immune abnormalities. Therefore, to be able to find out, it is necessary to do the initial research with emphasis on the activity of macrophages from TB-DM patients.

This study is aimed to analyze macrophage activity (phagocytic, intracellular killing and TNF$\alpha$ synthesis) from TB-DM patients, by identifying peripheral blood mononuclear cells (PBMC) of TB-DM patients.

\section{METHODS}

DM patients were obtained from the TB patients who have a history of $\mathrm{DM}$ and have a blood pressure more than 140/90mmHg. TB patients were recruited from the hospitals patients that have BTA $(+)$ and chest X-ray $(+)$. Healthy individu were the individu who have BTA (-) and chest X-ray (-). There were 18 samples of DM patients, TB patients, and healthy individu were recruited for this study, and all of the samples was signed the informed consents.

Ethical clearance was provided by Gadjah Mada University (EC numb. 36/EC/2.08.16). Most of the laboratory works were performed in Faculty of Medicine GadjahMada University.

\section{PBMC's Isolation (Peripheral Blood Mononuclear Cells)}

As much as $20 \mathrm{~mL}$ of peripheral blood was taken from TB-DM patients and continued with defibrination.

Ten milliliters of RPMI1640 medium (SigmaAldrich Inc., R0883)were added (RPMI 1640 medium without HI-PHS/Heat Inactivated Pooled Human Serum supplementation), then each $5 \mathrm{~mL}$ were moved into a tube containing $3 \mathrm{~mL}$ FicollHistopaque (Sigma-Aldrich Inc., 10771), then centrifuged. ${ }^{12,13,14}$ Supernatant layer was discarded, the pellet was rinsed and added with $4000 \mu 1$ RPMI $1640(+)$ medium (heated RPMI 1640 medium supplemented with $10 \%$ inactivated human serum with $56^{\circ} \mathrm{C}$ for 30 minutes/HI-PHS/Heat Inactivated Pooled Human Serum), and then continued by mix pipetting. Monocyte viability was determined using tryphan blue exclusion $(\geq 95 \%){ }^{16}$ Monocyte percentage was determined using Giemsa stain on the smear of centrifuge result. Monocyte $\left(10^{5} / \mathrm{mL}\right)$ were cultured in a 24-wells tissue culture plate covered by coverslip and then it was added with RMPI 1640 medium, 7,2 pH, contains $25 \mathrm{mM}$ HEPES and L-glutamine without serum and antibiotic. $^{16}$

\section{Opsonization of Mycobacterium tuberculosis}

An ose of platinum $\left(10^{6} \mathrm{CFU} / \mathrm{mL}\right)$ of $M$. tuberculosis H37Rv ATCC $27294^{\mathrm{T}}$ (signal) strain (derived from cultures of tuberculosis laboratory, Tropical Disease Centre Institute, Airlangga University)were inserted aseptically into screw cap tube containing $4000 \mu \mathrm{L}$ of Middlebrook $7 \mathrm{H} 9$ liquid medium and $\pm 6-7$ bead glass and homogenous vortex, then $4000 \mu \mathrm{L}$ suspension was added and centrifuged. Supernatant was discarded, the pellet was set aside and rinsed with $5000 \mu \mathrm{l}$ sterile PBS for 3 times. Pellet again was set aside and $4000 \mu 1$ RPMI 1640 (-) medium and $4000 \mu 1$ PHS/Pooled Human Serum were added. Next step is suction spray approximately 10 times with $26 \mathrm{G}$ tuberculin syringe and incubated at $37^{\circ} \mathrm{C}$ containing 5\% $\mathrm{CO}_{2}$ for 20 minutes then centrifuged. The supernatant layer was discarded, the pellet was rinsed with $5000 \mathrm{~mL}$ of sterile PBS for 3 times, then $4000 \mathrm{~mL}$ RPMI 1640 medium (+) were added.

\section{Co-Culture of Macrophage and M. tuberculosis}

On the $7^{\text {th }}$ day, the macrophage cells culture was added by $10^{6} \mathrm{CFU} / \mathrm{mL}$ suspension of opsonized- $M$. tuberculosis strain $\mathrm{H} 37 \mathrm{Rv}$ ATCC $27294 \mathrm{~T}$, and then it was incubated at $37^{\circ} \mathrm{C}$ with air containing 5\% $\mathrm{CO}_{2}$ for 24 hours, 48 hours, 7 times 24 hours and 14 times 24 hours. ${ }^{13,15,17}$ 


\section{Macrophage Activity Tests and TNF-a Levels Measurement}

Coverslip on the base of a 24-wells tissue culture plate was aseptically rinsed with sterile PBS for 5 times.

Sixty $\mu \mathrm{l}$ supernatant was taken from 24-well tissue culture plate aseptically, then test by used an ELISA kit (88-7346-76, Ready-SETGo!,eBioscience, DTA00C). TNF- $\alpha$ levels was measured by ELISA reader.

Coverslip base of a 24-well tissue culture plate was scraped to harvest the macrophage, then shaken well by mix pipetting, $200 \mu \mathrm{L}$ were taken and transferred into eppendorf tubes. An $800 \mathrm{~mL}$ sterile PBS were added and centrifuged. Supernatant layer was discarded, the pellet was set aside, added with $1000 \mathrm{~mL}$ of sterile distilled water, incubated for 30 minutes at $4{ }^{\circ} \mathrm{C}$ and then vortex for 5 minutes macrophage are lysis and intracellular $M$. tuberculosis free from macrophage.

Thirty $\mu \mathrm{l}$ was taken from, dropped on a solid Middlebrook $7 \mathrm{H} 10$ agar medium, and incubated at $37^{\mathrm{O}} \mathrm{C}$ with $5 \% \mathrm{CO}_{2}$ level for 7 days, 10 days, and 14 days to determine the number of bacteria surviving, not digested by macrophages; with counting colonies grown per $\mathrm{ml}(\mathrm{CFU} / \mathrm{ml}){ }^{18}$

\section{CFU Measurement}

The number of $M$. tuberculosis colonies which can still grow on solid Middlebrook $7 \mathrm{H} 10$ agar medium are counted as CFU/ml of the 7th, 10th, and 14 th day.

\section{Data Analysis}

The data came from the result of the macrophage microscopic observation of the TBDM patients, viable $M$. tuberculosis colony growth, and the TNF- $\alpha$ levels secreted by macrophages by used statistic test.

\section{RESULTS}

The study results showed that the mononuclear cells in the buffy coat layer from the peripheral blood of the TB-DM patients consists of monocytes and lymphocytes. Monocytes of TBDM patients which have matured into macrophages within 7 days were co-cultured with M. tuberculosis (in vitro). The results of microscopic observation from macrophages of TBDM patients showed that there is less number of ingested $M$. tuberculosis(red) and less formation of vacuoles and giant cell macrophages (blue) ) (Figure 1).

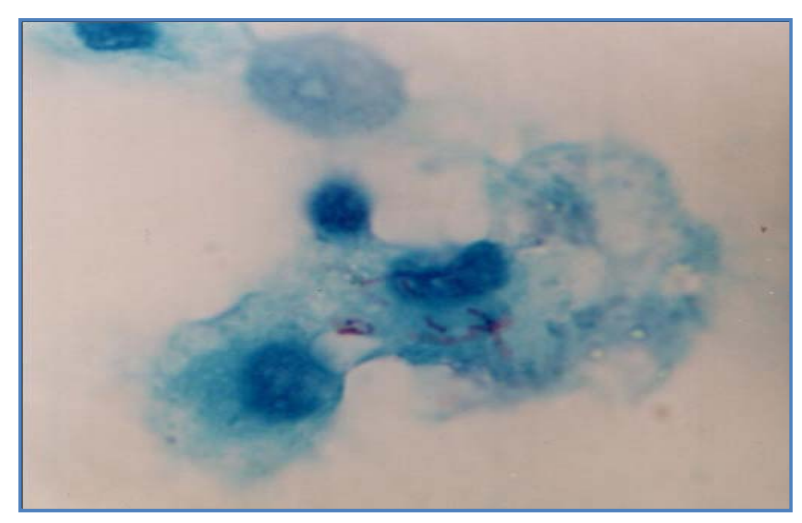

Figure 1.Macrophages of TB-DM patients ingested M. tuberculosis (NIKON Eclipse E600 light microscope, magnification of $1700 \mathrm{X}$ ).

TNF- $\alpha$ levels secreted by macrophages of TBDM patients, TB patients non-DM, and healthy individuals can be seen in Table 2 .

Based on Table 2, the average levels of TNF- $\alpha$ secreted by macrophages of TB-DM patients, TB patients without DM, and healthy individu can be seen as shown in Figure 2 .

Average of viable $M$. tuberculosis colonies calculated as $\mathrm{CFU} / \mathrm{ml}$ on day 7 th, 10 th, and $14^{\text {th }}$ can be seen in Figure 3.

\section{DISCUSSIONS}

Approximately $10-20 \%$ buffy coat is consisted of monocytes, the rest were lymphocytes. ${ }^{19}$ Buffy coat is located between the upper layer (plasma) and the bottom layer (erythrocytes and granulocytes sediment) from PBMCs of TB-DM patients, consisting monocytes and lymphocytes. TB-DM patients' monocytes which had matured into macrophages within 7 days appears as large rounded cells with regularly spherical nucleus resembling kidney/horseshoe (horseshoe-shaped), have cell wall protrusions and large cytoplasm, and also macrophages showing some presence of giant cells. While lymphocytes appeared as having large nuclei, round shape, heterochromatin features with a large nucleus-cytoplasm ratio. ${ }^{11,12,19,20}$ This study eliminated the presence of the lymphocytes using the PBS rinsing method in order to avoid the bias results from the process of intracellular killing because the lymphocytes have a role in producing IFN- $\gamma$ as a mediator of macrophage activation.

Additionally, this study carried out co-culture between macrophages and $M$. tuberculosis (in vitro). This was intended to provide the bacteria to invade macrophages in order to allow the phagocytosis process to take place. M. tuberculosis 
invasion process in macrophages can take place after an opsonization ${ }^{21,22}$ followed by binding and uptake of the complement receptors (CR1, CR3, and CR4), ${ }^{21,23}$ mannose receptor, surfactant, and Toll-Like Receptors (TLR). The interaction between opsonized $M$. tuberculosis with macrophages membranes can lead to ingestion/ engulfment and metabolic burst, which characterized by an increase in oxygen consumption $\left(\mathrm{O}_{2}\right)$, production of superoxide anion $\left(\mathrm{O}_{2}{ }^{-}\right)$, and hydrogen peroxide $\left(\mathrm{H}_{2} \mathrm{O}_{2}\right)$.

The process of ingestion/ engulfment begins with the introduction of $M$. tuberculosis by macrophages receptors, membranes of macrophages will surround the bacteria in a circle (zipper mechanism), thus the bacteria are in the phagosome. ${ }^{21}$ Phagosome then undergoes maturation and then fuse with lysosomes to form phagolysosome, ${ }^{21}$ an organelle with antimicrobial component and acidic $\mathrm{pH}(\mathrm{pH} \sim 6,2)$.

Macrophages from the activated TB patients are more effective in performing the phagosome and lysosome fusion. In addition, macrophages are also more effective in producing oxygen radicals, $\mathrm{NO}$, and various antimicrobial molecules ${ }^{26-28}$ and may increase respiratory burst, ROI production, RNI, and TNF- $\alpha$ releasing. ${ }^{29,30}$

Macrophages of TB-DM patients are activated macrophages, but because the TB-DM patients have defects in their immune cells, the macrophages become less activated. Furthermore, macrophages in DM patients have disruption of chemotaxis, phagocytosis, and antigen presenting phagocytes against $M$. tuberculosis. This defect cannot be resolved with insulin therapy. ${ }^{14}$ Patients with poorly controlled DM will upset the phagocytosis, especially if it has been in an acidosis state. This phagocytosis disruption is due to the intrinsic defect of the PMN. ${ }^{12}$

This results showed that there were decreased production of TNF- $\alpha$ in pulmonary tuberculosis patients with DM rather than non-diabetic TB patients. It is according to the literature mentioned that Th- 1 response levels, IFN- $\gamma$, as well as the production of IL- $1 \beta$ and IL- 6 are more profound in pulmonary tuberculosis patients with DM rather than non-diabetic TB patients. Decreased production of IFN- $\gamma$ was more significant in patients with pulmonary tuberculosis with uncontrolled diabetes than in patients with controlled DM TB. This IFN- $\gamma$ production will return to normal within six months, either in patients with pulmonary TB alone or pulmonary TB patients with uncontrolled DM, but will continue to decline in pulmonary TB patients with uncontrolled DM. Also, there were changes in pulmonary vascular and alveolar oxygen tension that aggravated the patients' condition. ${ }^{4,23,33}$

TNF- $\alpha$ proved to be effective in eliminating $M$. tuberculosis, this cytokine will increase the phagocytosis capability and induces apoptosis of infected macrophages. Both TNF- $\alpha$ and IFN- $\gamma$ will increase the intracellular killing macrophages process by producing reactive NO. TNF- $\alpha$ also plays a role in maintaining granuloma integrity, which formed when macrophages infected by $M$. tuberculosis. Macrophages that are unable or only slightly expresses TNF- $\alpha$ would be very easily infected and difficult to eliminate $M$. tuberculosis.

The presence of viable $M$. tuberculosis showed that this bacteria is able to escape and survive the macrophage phagocytosis and intracellular killing macrophage. Immune cell defects, fewer macrophages activation, and decreased in phagocytic capability in patients with TB-DM can support the viability of $M$. tuberculosis. In accordance with these results, it is known that there are more viable $M$. tuberculosis in TB-DM patients' macrophages as shown in Figure 3.

M. tuberculosis can also produce lipoarabinomannan (LAM), which can inhibit the activation of macrophages and is an important virulence determinant of these bacteria. LAM can inhibit the functions induced IFN- $\gamma$ thus disrupts the microbicidal function of macrophages, inhibiting the activity of protein kinase $\mathrm{C}$, and TNF- $\alpha$ evocation. ${ }^{34}$

\section{CONCLUSIONS}

Macrophages of TB-DM patients are less activated macrophages, where there are disturbances in its phagocytosis capability (due to the intrinsic defect of the PMN) and its phagocytes antigen presenting toward $M$. tuberculosis. These are shown in the results, that the TB-DM macrophages secreted low levels of TNF- $\alpha$ and there are more numbers of viable $M$. tuberculosis. 
Table 2. TNF- $\alpha$ levels secreted by macrophages of TB-DM patients, TB patients without DM, and healthy individu

\begin{tabular}{|c|c|c|c|c|c|c|c|c|c|c|c|c|c|c|c|c|c|c|}
\hline \multirow{5}{*}{ TNF- $\alpha$} & \multicolumn{6}{|c|}{$\begin{array}{c}\text { TB-DM patients } \\
(\mathrm{pg} / \mathrm{ml})\end{array}$} & \multicolumn{6}{|c|}{$\begin{array}{l}\text { TB patients without DM } \\
\text { (pg/ml) }\end{array}$} & \multicolumn{6}{|c|}{$\begin{array}{c}\text { Healthy Individu } \\
(\mathrm{pg} / \mathrm{ml})\end{array}$} \\
\hline & 1 & 2 & 3 & 4 & 5 & 6 & 1 & 2 & 3 & 4 & 5 & 6 & 1 & 2 & 3 & 4 & 5 & 6 \\
\hline & 78 & 97 & 81 & 103 & 91 & 81 & 101 & 132 & 98 & 111 & 123 & 132 & 89 & 103 & 86 & 127 & 99 & 101 \\
\hline & 86 & 98 & 97 & 87 & 75 & 103 & 111 & 143 & 117 & 97 & 121 & 98 & 98 & 107 & 98 & 113 & 97 & 97 \\
\hline & 93 & 89 & 101 & 81 & 97 & 97 & 96 & 118 & 81 & 134 & 117 & 131 & 100 & 97 & 99 & 136 & 103 & 131 \\
\hline
\end{tabular}

Table 3.Mean and standard deviation of TNF- $\alpha$ levels secreted by macrophages of TB-DM patients, TB patients without DM, and healthy individu

\begin{tabular}{|c|c|c|c|c|c|c|c|c|c|c|c|c|c|c|c|c|c|c|}
\hline \multirow{7}{*}{$\frac{\text { Mean }}{\text { SD }}$} & \multicolumn{6}{|c|}{$\begin{array}{c}\text { TB-DM patients } \\
(\mathrm{pg} / \mathrm{ml})\end{array}$} & \multicolumn{6}{|c|}{$\begin{array}{c}\text { TB patients without DM } \\
(\mathrm{pg} / \mathrm{ml})\end{array}$} & \multicolumn{6}{|c|}{$\begin{array}{c}\text { Healthy Individu } \\
(\mathrm{pg} / \mathrm{ml})\end{array}$} \\
\hline & 1 & 2 & 3 & 4 & 5 & 6 & 1 & 2 & 3 & 4 & 5 & 6 & 1 & 2 & 3 & 4 & 5 & 6 \\
\hline & $\underline{41.2}$ & 48.1 & $\underline{43.6}$ & 44.1 & $\underline{41.5}$ & $\underline{48.1}$ & 45.1 & 46.7 & $\underline{47.1}$ & 44.8 & $\underline{46.5}$ & 47.2 & $\underline{44.8}$ & 42.6 & 49.1 & 47.6 & 46.5 & $\underline{44.0}$ \\
\hline & $\overline{2.2}$ & $\overline{2.3}$ & $\overline{2.5}$ & $\overline{2.7}$ & $\overline{2.1}$ & $\overline{2.3}$ & $\overline{2.0}$ & $\overline{2.3}$ & $\overline{2.3}$ & $\overline{2.1}$ & $\overline{2.1}$ & $\overline{2.5}$ & 2.1 & $\overline{2.4}$ & $\overline{2.6}$ & $\overline{2.4}$ & $\overline{2.1}$ & 2.5 \\
\hline & 46.2 & 41.3 & 42.1 & 44.3 & 47.3 & 41.3 & 48.3 & 48.1 & 49.1 & 44.5 & 42.8 & 42.7 & 42.8 & 41.3 & 44.8 & 41.5 & 44.1 & 42.6 \\
\hline & $\underline{44.0}$ & 43.1 & 41.2 & 43.6 & $\underline{42.3}$ & $\underline{42.6}$ & $\underline{47.6}$ & $\underline{47.6}$ & $\underline{44.8}$ & 45.7 & $\underline{42.8}$ & $\underline{47.3}$ & 44.1 & $\underline{43.6}$ & $\underline{41.2}$ & 45.1 & 43.1 & $\underline{41.5}$ \\
\hline & $\overline{2.5}$ & $\overline{2.3}$ & $\overline{2.2}$ & $\overline{2.5}$ & $\overline{2.6}$ & $\overline{2.4}$ & $\overline{2.6}$ & $\overline{2.4}$ & $\overline{2.3}$ & $\overline{2.4}$ & $\overline{2.6}$ & $\overline{2.3}$ & 2.7 & $\overline{2.5}$ & $\overline{2.2}$ & $\overline{2.0}$ & $\overline{2.3}$ & 2.1 \\
\hline
\end{tabular}

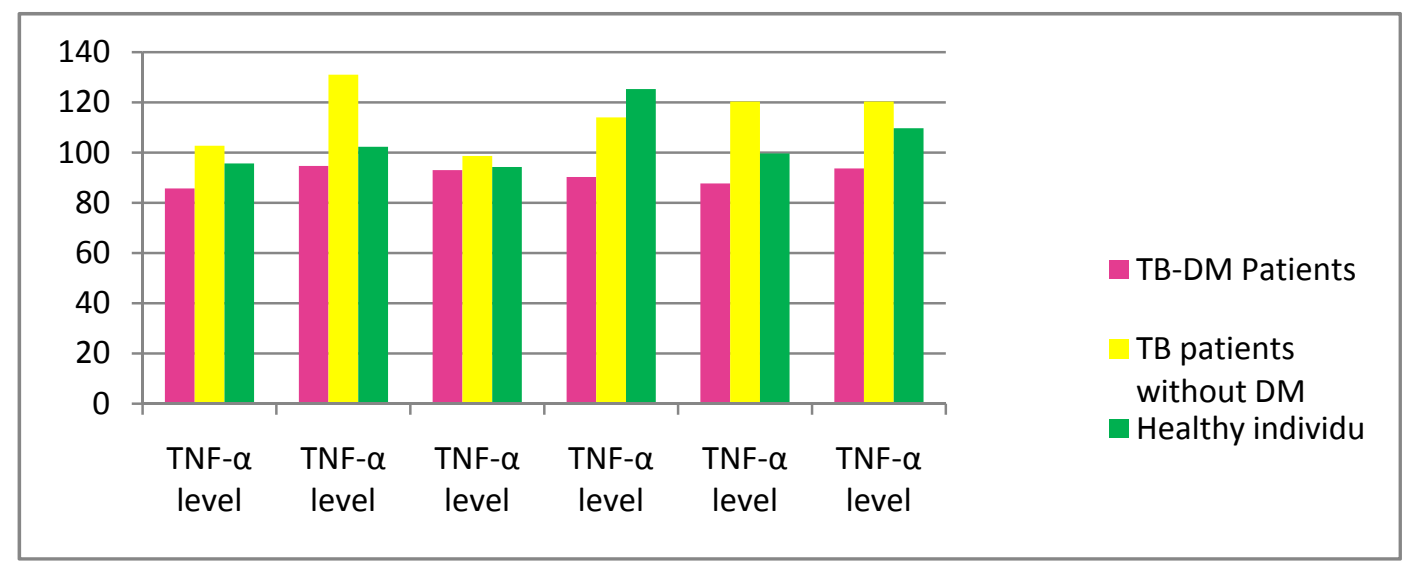

Figure 2. Average of TNF- $\alpha$ levels secreted by macrophages of TB-DM patients, TB patients non-DM, and healthy individuals.

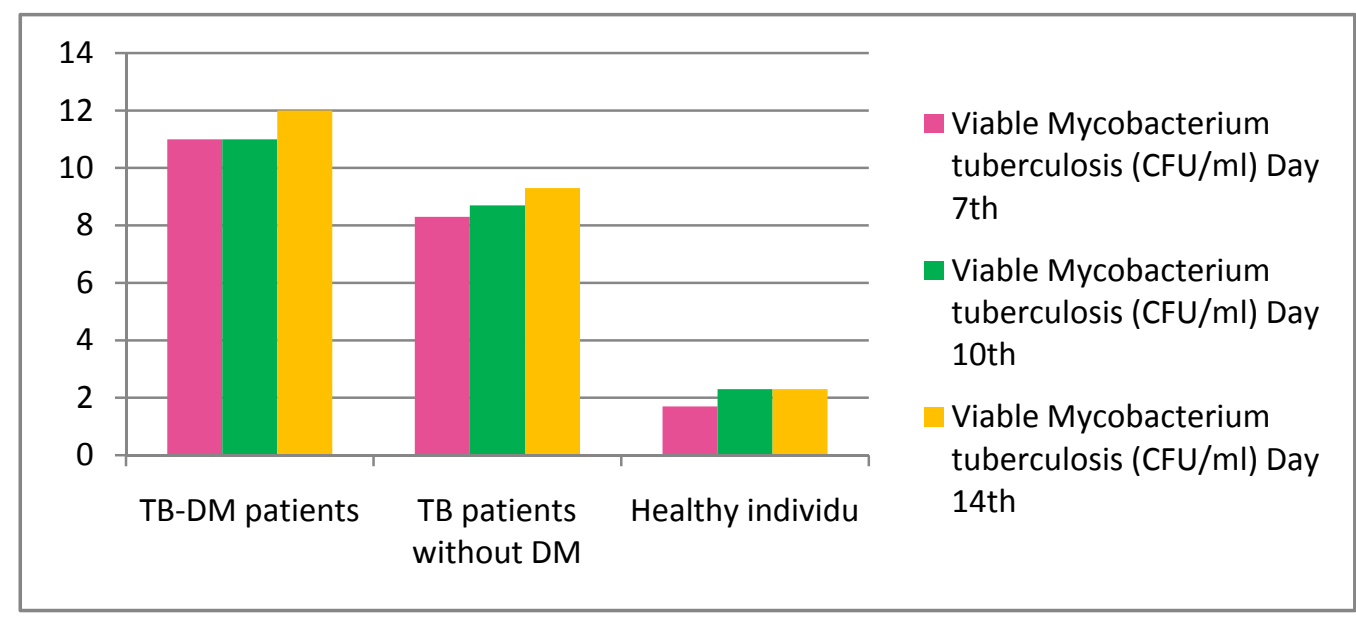

Figure 3. Average number of viable M. tuberculosis colonies (CFU / ml). 


\section{REFERENCES}

1. International Diabetes Federation. Diabetes and tuberculosis.2013.

http://www.idf.org/diabetes/5e/diabetes-andtuberculosis.

2. World Health Organization. 2010. Global tuberculosis control. Geneva: World Health Organization.

3. Perhimpunan Dokter Paru Indonesia (PDPI). 2011. Tuberkulosis: Pedoman diagnosis dan penatalaksaan di Indonesia. Jakarta: Indah Offset Citra Grafika.

4. Guptan A., Shah A. 2000. Tuberculosis and diabetes: An appraisal. Ind. J. Tub. 47(3):2-7

5. Jeon C. Y., Murray M. B. 2012. Diabetes mellitus increases the risk of active TB: a systematic review of 13 observational studies. PLoS Med.

6. Yamashiro S., Kawakami K., Uezu K., Kinjo T., Miyagi K., Nakamura K. 2011. Lower expression of Th1-related cytokines and inducible nitric oxide synthase in mice with streptomycin-induced diabetes mellitus infected with Mycobacterium tuberculosis. Clin Exp Immunol. 139:57-64.

7. Baratawidjaja K. G. 1996. Imunologidasar. Jakarta. Fakultas Kedokteran Universitas Indonesia.

8. Zhang X.,Goncalves R., Mosser D.M. 2008.The Isolation and characterization of murine macrophages. CurrProtocImmunol. Chapter : Unit-14.1.

9. Wang C., Yu X., Cao Q., Wang Y., Zheng G., Tan T.K., ZhaoH., Zhao Y., Wang Y., Harris D.C.H. 2013. Characterization of murine macrophages from bone marrow, spleen and peritoneum.BMC Immunol. 14:6.

10. Italiani P., Boraschi D. 2014. From monocytes to M1/M2 Macrophages: Phenotypicalvs. Functional differentiation. Front Immunol. 17(5):514

11. WangW., Wang J., Dong S.F., Liu C.H., Italiani P., Sun, S.H., Xu J., Boraschi D., Ma S., Qu D. 2010. Immunomodulatory activity of andrographolide on macrophage activation and specific antibody response. Acta PharmacolSin.31(2):191-201.

12. Vega V.L., Charles W., Alexander L. E. C. 2011. Rescuing of deficient killing and phagocytic activities of macrophages derived from non-obese diabetic mice by treatment with geldanamycin or heat shock: potential clinical implications. Cell stress chaperones 16(5):57381.

13. Espinoza-JimenezA., Peon A.N., Terrazas, L.I. 2012. Alternatively activated macrophages in types 1 and 2 diabetes. Mediatorsinflamm.815953.

14. Dao D. N. 2010. Mycobacterium tuberculosis lipoarabinomannan inducer apoptosis and interleukin-12 production in macrophages. Infection and immunity journals. 72(4): 2067-
74.

15. Orsi R. 2010. Immunomodulatory action on macrophage activation. J Venom Anim Toxin. 6(2): 205-19.

16. LiuH.F., Zhang H.J., Hu Q.X., Liu X.Y., Wang Z.Q., Fan J.Y., Zhan M., Chen F.L. 2012. Altered polarization, morphology, and impairedinnate immunitygermane to resident peritoneal macrophages in mice with long-term type 2 diabetes. J Biomed Biotechnol.867023.

17. Parsa R., Andresen P., Gillett A., Mia S., Zhang X.M., Mayans S.,Holmberg D., Harris R. 2012. Adoptive transfer of immunomodulatory M2 macrophages prevents type 1 diabetes in NOD mice. Diabetes61(11):2881-92.

18. Bellanti A. J. 1993. Imunologi. Jakarta. Gadjah Mada University Press.

19. Lopez D.,and Handle-Fernandez M. E. 2010. Isolation of macrophages from tissue, fluids, and immune response sites. Department of microbiology and immunology. Miami. USA.

20. Diggs, Sturn, and Bell. 2011. The morphology of human blood cells. $8^{\text {th }}$ ed. Tennesee University. Memphis. Abbot Publisher.

21. Turgeon M. L. 2009. Clinical hematology theory and procedures. $6^{\text {th }}$ ed. USA: Lippincott Williams \& Wilkins.

22. Banki A., Jenei P., and Richards G. 2010. Mycobacterium tuberculosis and its host cell, the macrophage.

http://www.sp.uconn.edu/ terry/Spring96/Web TB2/Groups/Group5/Final.html.

23. Crevel V., Tom H.., Ottenhoff, Jos W. M. 2012. Innate immunity to Mycobacterium tuberculosis. Clinical microbiology review. P. 294-309.

24. Collins H. L. and Kaufmann S. H. E. 2011. The many faces of host responses to tuberculosis, pathogenesis, protection, and control. Edited by Bloom B. Washington DC: ASM Press. pp $389-416$.

25. Schluger N. W. 2001. Recent advances in our understanding of human host responses to tuberculosis. Associate professor of medicine and public health, Columbia University College of Physicians and Surgeon, New York, USA. http://respiratory-research.com/content/2/3/157.

26. Brookes R. H., Pathan A. A., McShane H., Hensmann M., Price D. A. and Hill A. V., 2013. CD8+ T-Cell Mediated Suppression of Intracellular Mycobacterium tuberculosis Growth in Activated Human Macrophages. EUR J.Immunol. 33(12):3293 - 302.

27. Wigginton J. and Kirschner D., 2011. A Model to Predict Cell-Mediated Regulatory Mechanisms During Human Infection with Mycobacterium tuberculosis. Department of Microbiology and Immunology. University of MichiganMedical School, Ann Arbor.

28. Paulnock D. M., 2010. Macrophages: A Practical Approach. USA. Oxford University 
Press.

29. Dao D. N. et al., 2013. Mycobacterium tuberculosis Lipomannan Inducer Apoptosis and IL-12 Production in Macrophages. Dalam Infection and Immunity Journals. Vol 72 no. 4 p 2067 - 2074.

30. Abbas A. K., Andrew H. and Pober J. S., 2010. Cellular and Molecular Immunology. $4^{\text {th }}$ Edition. Philadelphia: WB. Saunders Company. pp. $352-354$.

31. Petrunov B., Nenkov P., and Shekerdjisky R. 2007. The role of immunostimulants in immunotherapy and immunoprophylaxis. Bulgaria. National center of infectious and parasitic disease. BulBio-NCIPD Natsim Ltd.

32. Sanusi S., 2004. Diabetes mellitus dan tuberculosis paru. J. Med Nus. 25: 1-5.

33. WidjajatiLaely. 2012. Manfaat dan khasiat bunga sepatu.

34. Debasish M., Bhattacharyya A., and Basu J., 2011.Lipoarabinomannan from Mycobacterium tuberculosis Promotes Macrophage Survival by Phosphorylating Bad through a Phosphatidylinositol 3-Kinase/Akt Pathway. Department of Chemistry, Bose Institute, Acharya Prafulla Chandra Road, Calcutta, India .Chandra Road, Calcutta, India. 